\title{
Renormalization Group study of the Sliding Luttinger liquids
}

\author{
Germán Sierra \\ Instituto de Matemáticas y Fúsica Fundamental, C.S.I.C., Madrid, Spain.
}

(Dated: November 10, 2018)

\begin{abstract}
We derive the RG-flow equations of the sliding Luttinger liquid perturbed by charge-densitywave $(\mathrm{CDW})$ and superconducting $(\mathrm{SC})$ operators. Using them we study the phase diagram of an array of XXZ spin chains coupled by Ising terms. In the weak coupling regime we find a new class of non-gaussian and unstable fixed points whose existence is due to a balance between the CDW, SC and sliding Luttinger couplings.

PACS numbers: 71.10.Hf, 75.10.Jm, 75.30.Gw, 74.20.Mn
\end{abstract}

The Luttinger liquid (LL) is the key concept to describe interacting electrons in one dimension. The collective nature of the excitations, the spin-charge separation and the power law behaviour of correlators with anomalous exponents are some of its distintive feautures, in contrast to the Fermi liquid theory [1]. Much of the theoretical activity in the last years has been devoted to the search of non-Fermi liquid theories in dimensions higher than one, specially in $2 \mathrm{D}$, due to its possible connection with the high- $T_{c}$ superconductors and another strongly correlated systems.

A natural path to realize a non-Fermi liquid has been to couple arrays of 1D Luttinger liquids forming ladders and 2D planes. However the general consensus has been, until recently, that 2D arrays of LL's are unstable to the formation of crystal, superconducting or 2D Fermi liquid states [2]. An alternative to these "no-go theorems" has been proposed lately using the concept of sliding Luttinger liquid (SLL), also called smectic non-Fermi liquid

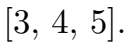

These works were partially motivated by the Anderson's proposal of confinement of excitations in the Luttinger liquids [6] and have a classical analogue in the stacks of coupled 2D XY models coupled by gradient interactions [7]. The SLL model may also be relevant to the stripe phases of the Quantum Hall effect and the cuprates.

The sliding Luttinger liquid is the fixed point of a gaussian Hamiltonian which treats on equal footing the individual Luttinger Hamiltonians of the stripes and the density-density and current-current interstripe interactions [3, 4]. Using bosonization techniques one can regard the SLL as a set of decoupled LL's characterized by a sound velocity $v\left(q_{\perp}\right)$ and a Luttinger coupling $K\left(q_{\perp}\right)$ which depend on the tranverse momentum $q_{\perp}$ across the stripes. The gaussian nature of the SLL fixed point allows a simple derivation of the scaling dimensions of the single particle (SP), charge density (CDW) and superconduct- ing (SC) order parameters, in terms of the SLL function $K\left(q_{\perp}\right)$ 3, , 1 . These scaling dimensions have been used to study the stability of the SLL under various perturbations, finding rich phase diagrams where the SLL phase survives in the vicinity of $\mathrm{CDW}, \mathrm{SC}$ and Fermi liquid phases [3, 4, 5]. The perturbative RG analysis performed in the latter references take into account the running of the coupling constants of the perturbations to first order, while the Luttinger functions $K\left(q_{\perp}\right)$ and $v\left(q_{\perp}\right)$ stay constant. It is however well known that in certain situations, as in the presence of marginal perturbations, one has to consider in addition the renormalization of the Luttinger parameters in a RG à la Kosterlitz-Thouless (KT) [8]. This is for example the case of the Hubbard model at half filling where the Umklapp operator becomes marginally relevant for a repulsive Hubbard constant, leading to a charge gap in the low energy spectrum.

The aim of this Letter is to derive the RG equations of the Sliding Luttinger liquid for the coupling constants and the SLL functions to second order in the couplings, and study some of their consequences in a model consisting of arrays of spinless 1D Hamiltonians coupled by density-density interactions. The latter model has been treated in the past with bosonization [9], mean field [10] and variational methods 11, which predict the existence of large regions in parameter space where the phase is either CDW-like or XY-like, corresponding respectively to the smectic crystal and the smectic metallic phases of references [3, 何.

On more general grounds we also analyse the stability of the spinless SLL under marginal CDW and SC perturbations, reaching the conclusion that when one of these perturbations is irrelevant the other one is relevant and hence the SSL is an unstable fixed point. We also find new unstable fixed points if two conditions are satisfied: i) when the CDW coupling constants are minus the superconducting ones, which guarantees the freezing of the SLL parameters, and ii) when the running of the 
CDW and SC parameters to first order, which is given by their scaling dimension, is cancelled out by the second order term. The mechanism involved here is similar to the one giving rise to the Wilson-Fisher fixed point in dimensions $d<4[12]$. From a more mathematical viewpoint the manifold of these non gaussian fixed points are closely related to the target manifolds that appear in 5 dimensional supergravity theories $[13]$.

Let us consider an array of $N$ spinless Luttinger stripes with phase fields for the density fluctuations $\phi_{a}(a=$ $1, \ldots, N)$ and euclidean Lagrangian

$$
\mathcal{L}_{0}=\sum_{a=1}^{N} \frac{K_{0}}{2}\left[\frac{1}{v_{0}}\left(\partial_{t} \phi_{a}\right)^{2}+v_{0}\left(\partial_{x} \phi_{a}\right)^{2}\right]
$$

where $K_{0}$ is the inverse of the standard Luttinger parameter ( $K_{0}>1$ for repulsion) and $v_{0}$ is the sound velocity which we scale to 1 . The charge density fluctuations $j_{0}^{a}$ and the charge currents $j_{1}^{a}$ are given by the bosonization equation $j_{\mu}^{a}=\frac{1}{\pi} \epsilon_{\mu \nu} \partial^{\nu} \phi_{a}$. Hence the density-density and current-current interactions among the stripes are also quadratic in the derivatives of the bosonic fields and, together with (11), define the sliding Luttinger Lagrangian [3, 幽

$$
\mathcal{L}_{S L L}=\frac{1}{2} \sum_{a, b=1}^{N}\left[\partial_{t} \phi_{a} K_{a, b}^{J} \partial_{t} \phi_{b}+\partial_{x} \phi_{a} K_{a, b}^{\rho} \partial_{x} \phi_{b}\right]
$$

where $K_{a, b}^{J, \rho}=\delta_{a, b} K_{0}+\bar{K}_{a, b}^{J, \rho}$ are $N \times N$ matrices whose off-diagonal elements are given by the interstripe currentcurrent and density-density interactions. The SLL model can alternatively be formulated in the dual variables $\theta_{a}$, which are the phase fields of the superconducting fluctuations. The SLL Lagrangian (2) becomes [3, 4, 5]

$$
\mathcal{L}_{S L L}=\frac{1}{2} \sum_{a, b=1}^{N}\left[\partial_{t} \theta_{a}\left(K_{\rho}^{-1}\right)_{a, b} \partial_{t} \theta_{b}+\partial_{x} \theta_{a}\left(K_{J}^{-1}\right)_{a, b} \partial_{x} \theta_{b}\right]
$$

where $K_{J, \rho}^{-1}$ are the inverse matrices of $K^{J, \rho}$. Both eqs.(2) and (3) exhibit the smectic or sliding symmetries $\phi_{a} \rightarrow \phi_{a}+\alpha_{a}$ and $\theta_{a} \rightarrow \theta_{a}+\beta_{a}$, where $\alpha_{a}$ and $\beta_{a}$ are constants, which prevents the lock in of the chargedensity-wave and superconducting order parameters of the individual stripes [3, 4]. Assuming periodic boundary conditions across the stripes and translational invariance along them, one can perform the Fourier transform: $\phi_{a}=\frac{1}{\sqrt{N}} \sum_{q_{\perp}} e^{i q_{\perp} a} \phi_{q_{\perp}}, \quad K^{J, \rho}\left(q_{\perp}\right)=\sum_{q_{\perp}} e^{i q_{\perp} a} K_{1,1+a}^{J, \rho}$ in order to bring the SLL Lagrangian (2) into the form

$$
\mathcal{L}_{S L L}=\frac{1}{2} \sum_{q_{\perp}} K\left(q_{\perp}\right)\left[\frac{1}{v\left(q_{\perp}\right)}\left|\partial_{t} \phi_{q_{\perp}}\right|^{2}+v\left(q_{\perp}\right)\left|\partial_{x} \phi_{q_{\perp}}\right|^{2}\right]
$$

where $K\left(q_{\perp}\right)=\sqrt{K^{J}\left(q_{\perp}\right) K^{\rho}\left(q_{\perp}\right)}$ is the (inverse) Luttinger parameter and $v\left(q_{\perp}\right)=\sqrt{K^{\rho}\left(q_{\perp}\right) / K^{J}\left(q_{\perp}\right)}$ is the sound velocity of the $q_{\perp}$-mode. In the dual variables $\theta_{q_{\perp}}$ the Lagrangian has the same form as eq.(44) with the replacement $K\left(q_{\perp}\right) \rightarrow 1 / K\left(q_{\perp}\right)$. The scaling dimensions of the various operators, given in references [3, 4], can be easily derived by observing that a generic vertex operator $V_{\boldsymbol{\beta}}^{\phi}=\exp \left(i \sum_{a} \beta_{a} \phi_{a}\right)$, factorizes into the product of vertex operators on the $q_{\perp}$-modes as follows

$$
\begin{aligned}
V_{\boldsymbol{\beta}}^{\phi} & =\prod_{0 \leq q_{\perp} \leq \pi} e^{i \sqrt{\frac{2}{N} \sum_{a} \beta_{a}\left(\cos \left(q_{\perp} a\right) \phi_{q_{\perp}}^{(1)}+\sin \left(q_{\perp} a\right) \phi_{q_{\perp}}^{(2)}\right)}} \\
\phi_{q_{\perp}} & =\frac{1}{\sqrt{2}}\left(\phi_{q_{\perp}}^{(1)}-i \phi_{q_{\perp}}^{(2)}\right), \quad\left(q_{\perp} \neq 0, \pi\right)
\end{aligned}
$$

where $\phi_{q \perp}^{(1,2)}$ are real scalar fields. The scaling dimension $\Delta_{\boldsymbol{\beta}}^{\phi}$ of $V_{\boldsymbol{\beta}}^{\phi}$ is given simply by the sum of the scaling dimensions of all the individual $q_{\perp}$-modes, namely

$$
\Delta_{\boldsymbol{\beta}}^{\phi}=\int_{q_{\perp}} \frac{1}{4 \pi K\left(q_{\perp}\right)}\left(\sum_{a} \beta_{a}^{2}+2 \sum_{a<b} \beta_{a} \beta_{b} \cos \left(q_{\perp}(a-b)\right)\right)
$$

where $\int_{q_{\perp}}=\int_{-\pi}^{\pi} \frac{d q_{\perp}}{2 \pi}$. Similary, a vertex operator in the dual variables, i.e. $V_{\boldsymbol{\beta}}^{\theta}=\exp \left(i \sum_{a} \beta_{a} \theta_{a}\right)$, has a scaling dimension $\Delta_{\boldsymbol{\beta}}^{\theta}$ given by the formula (6) with $K\left(q_{\perp}\right)$ replaced by its inverse. The interaction Lagrangian is given by the pair hopping (SC) and particle-hole (CDW) operators [3, 4],

$$
\begin{aligned}
\mathcal{L}_{i n t}= & \frac{1}{2} \int \frac{d^{2} x}{\left(2 \pi a_{0}\right)^{2}} \sum_{a, b}\left[g_{C D W}^{a, b} \cos \beta\left(\phi_{a}-\phi_{b}\right)\right. \\
& \left.+g_{S C}^{a, b} \cos \beta\left(\theta_{a}-\theta_{b}\right)\right]
\end{aligned}
$$

where $a_{0}$ is the lattice spacing and $\beta=\sqrt{2 \pi}$ for the charge modes of spin gapped systems [3], or $\beta=\sqrt{4 \pi}$ for spinless fermions [4]. In the latter references the stability of the perturbed SLL was studied in terms of the relevance or irrelevance of the $\mathrm{CDW}$ and $\mathrm{SC}$ operators (7), given by their scaling dimensions. There are however cases where one has to consider the renormalization of the functions $K\left(q_{\perp}\right)$ and $v\left(q_{\perp}\right)$, for example when the CDW and SC operators become marginal. The latter situation actually arises in the study of the coupled XXZ spin chain Hamiltonians via Ising and spin-pair-flipping terms [9, 11],

$$
\begin{aligned}
& H=\sum_{i=1}^{L} \sum_{a=1}^{N}\left[-\frac{J}{2}\left(S_{i, a}^{+} S_{i+1, a}^{-}+S_{i, a}^{-} S_{i+1, a}^{+}\right)+J_{z} S_{i, a}^{z} S_{i+1, a}^{z}\right. \\
& \left.+J_{z}^{\prime} S_{i, a}^{z} S_{i, a+1}^{z}+J_{X Y}^{\prime}\left(S_{i, a}^{+} S_{i+1, a}^{+} S_{i, a+1}^{-} S_{i+1, a+1}^{-}+\text {h.c. }\right)\right]
\end{aligned}
$$


This model can be Jordan-Wigner transformed into a spinless fermion Hamiltonian which, after bosonization, becomes at half filling [9],

$$
\begin{aligned}
& H=\sum_{a=1}^{N} \int d x\left[\frac{u}{2 K_{0}} \pi_{a}^{2}+\frac{u K_{0}}{2}\left(\partial_{x} \phi_{a}\right)^{2}+\frac{2 J_{z} a_{0}}{\left(2 \pi a_{0}\right)^{2}} \cos \sqrt{16 \pi} \phi_{a}\right. \\
& +\frac{J_{z}^{\prime} a_{0}}{\pi} \partial_{x} \phi_{a} \partial_{x} \phi_{a+1}+\frac{8 J_{X Y}^{\prime} a_{0}}{\left(2 \pi a_{0}\right)^{2}} \cos \sqrt{4 \pi}\left(\theta_{a}-\theta_{a+1}\right)+
\end{aligned}
$$

$$
\left.\frac{2 J_{z}^{\prime} a_{0}}{\left(2 \pi a_{0}\right)^{2}}\left(\cos \sqrt{4 \pi}\left(\phi_{a}+\phi_{a+1}\right)-\cos \sqrt{4 \pi}\left(\phi_{a}-\phi_{a+1}\right)\right)\right]
$$

where $u=J a_{0}\left(1+2 J_{z} /(\pi J)\right.$ and $K_{0}=1+2 J_{z} /(\pi J)$ for $\left|J_{z}\right|<<J$. The gaussian terms in (9) yield a SLL model (2) with

$$
K_{a, b}^{J}=\delta_{a, b} K_{0}, \quad K_{a, b}^{\rho}=\delta_{a, b} K_{0}+\frac{J_{z}^{\prime}}{\pi} \delta_{|a-b|, 1}
$$

where the time variable and the exchange couplings are measured in units of $u$ and $J$ respectively. In the weak coupling regime $\left|J_{z}\right|<<J$, the SLL function $K\left(q_{\perp}\right)$ is close to 1 . Consequently the intra-chain umklapp couplings $\cos \sqrt{16 \pi} \phi_{a}$ have dimension $d \sim 4$ and hence can be neglected, as it is the case of decoupled single chains. On the other hand the CDW, SC and Umklapp interchain couplings are marginal and one has to consider their running together with that of the SLL functions. To simplify matters we shall neglect in what follows the Umklapp term, which is anyway absent away from halffilling. The couplings constants in (7), which correspond to (9), are given by

$$
g_{C D W}^{a, b}=-2 J_{z}^{\prime} \delta_{|a-b|, 1}, \quad g_{S C}^{a, b}=8 J_{X Y}^{\prime} \delta_{|a-b|, 1}
$$

Eqs.(2) and (7) define a multicomponent sine-Gordon model which can be renormalized using operator product expansion (OPE) techniques 12. We shall further assume that the sound velocity of the modes is constant and set it to 1 , so that the hard disk regularization will be common to all the modes. The RG-flow equations for $K_{a, b}=K_{a, b}^{J, \rho}$ and $g_{C D W / S C, n}$ are given by ( up to second order in the $g^{\prime} s$ )

$$
\begin{aligned}
\frac{d K_{a, b}}{d s} & =\frac{\beta^{2}}{(4 \pi)^{3}}\left[F_{C D W}^{0} \delta_{a, b}-\left(g_{C D W}^{a, b}\right)^{2}\right. \\
& \left.-\sum_{c, d} K_{a, c}\left(F_{S C}^{0} \delta_{c, d}-\left(g_{S C}^{c, d}\right)^{2}\right) K_{d, b}\right] \\
\frac{d g_{X}^{a, b}}{d s} & =\left(2-\Delta_{X}^{a, b}\right) g_{X}^{a, b}-\frac{1}{4 \pi} F_{X}^{a, b} \quad(X=S C, C D W)
\end{aligned}
$$




$$
\frac{d x}{d s}=-y^{2}, \frac{d y}{d s}=-x y
$$

which are the well known Kosterlitz-Thouless RG eqs. of the XY model [8, 12. In this case the norm $\mu$ (17) is proportional to $x^{2}-y^{2}$, and it is conserved since the cubic term vanishes. The RG trayectories are hyperbolas of constant $\mu$ which end up at infinity provided $|y|>$ $x$. The latter inequality translates in the XXZ+Ising model (8) into the condition $\left|J_{z}^{\prime}\right|>-4 J_{z}$, which coincides with the one obtained in reference [9] for the existence of an AF-Ising phase. In the weak coupling regime of the $\mathrm{XY}$ model, which corresponds to the region $|y|<x$, the parameter $y$ flows to zero and $x$ flows to the line $x>0$ of SLL stable fixed points. The inclusion of the interstripe Luttinger parameter $k_{1}$ modifies the boundaries of the strong coupling region. Indeed performing the change of variables $x=-2 k_{0}+k_{1}$ and $y=\sqrt{6} g_{C D W, 1} /(4 \pi)$ one obtains from (15) the same KT eqs.(19), and the strong coupling regime is given now by $J_{z}^{\prime}>-17.798 J_{z}$ ( if $J_{z}^{\prime}>$ 0 ) and $-J_{z}^{\prime}>-1.798 J_{z}$ (if $J_{z}^{\prime}<0$ ). In all these cases a necessary condition to achieve a XY or SLL phase is to have a ferromagnetic intrachain coupling $J_{z}<0$, which agrees with the results of [4] concerning the proximity of the stable SLL to the isotropic ferromagnetic point where the boson stifness $K_{0}$ vanishes. To consider the effect on the higher order couplings we make the change of variables $x=-2 k_{0}$ and $y_{n}=g_{C D W, n} /(2 \pi)$, obtaining the eqs.

$$
\frac{d x}{d s}=-\sum_{n=1}^{M} y_{n}^{2}, \quad \frac{d y_{n}}{d s}=-x y_{n}-\frac{1}{2} \frac{\partial \mathcal{N}(y)}{\partial y_{n}}
$$

If the initial conditions are set to $x(0)=x, y_{1}(0)=$ $y, y(0)-0 \quad(1<n \leq M)$ we obtain numerically that the strong coupling regime arises when $\left|y_{1}\right|>A_{M}$ where $A_{M}$ converges exponentially to 0.83 in the limit $M \rightarrow \infty$. Hence the AF-Ising region of [9] gets enlarged by the inclusion of all the couplings generated by the RG, while SLL region diminishes. One can similarly add the effect of the $k_{n}$ parameters which change again the boundaries of the AF-Ising region.

Another interesting case is when there are both CDW and SC couplings. As can be seen seen from eqs. (15) the SLL fixed point, where $g_{C D W, n}=g_{S C, n}=0$, is unstable since their scaling dimensions are $\pm\left(2 k_{0}-k_{n}\right)$. However there is another fixed point if $g_{C D W, n}=-g_{S C, n}$ or $g_{C D W, n}=(-1)^{n+1} g_{S C, n}$ and

$$
\left(2 k_{0}-k_{n}\right) g_{C D W, n}=\frac{1}{4 \pi} \frac{\partial \mathcal{N}\left(g_{C D W}\right)}{\partial g_{C D W, n}}
$$

This equation has non trivial solutions for $g_{n}$ as functions of $k_{n}$. The fixed points we have analized turn out to be unstable. The non-gaussian nature of these fixed points recall the Wilson-Fisher fixed point, with $2 k_{0}-k_{n}$ playing the role of $\epsilon=4-D$. From a completely different viewpoint it is worth to mention that the mathematical structure underlying these fixed points, is very close to the one that appears in 5D-Supergravity theories [13], where the manifold of the scalar fields, that come in the Maxwell multiplets, is defined in terms of a cubic norm $\mathcal{N}$ similar to the one introduced in (16). This connection may help to study the properties of the new solutions. An interesting problem is to investigate the existence of stable non-gaussian SLL fixed points when $K\left(q_{\perp}\right)$ does not lie near 1 . This can be done starting from eqs. (12) rather than from their linearized version (15). Another issue is to include the effect of non constant $v\left(q_{\perp}\right)$. The generalization of our results to SLL's with charge and spin degrees of freedom is rather straightforward. Here too we expect the appearance of novel non-gaussian fixed points.

\section{Acknowledgments}

I would like to thank M.A. Martín-Delgado and J. Rodriguez for conversations. This work has been supported by the Spanish grant BFM2000-1320-C02-01.

[1] V.J. Emery, in "Highly Conducting one-dimensional solids", J. Dovrese et al. (Plenum, New York, 1979).

[2] H.J. Schulz, J. Phys. C 16, 6769 (1983).

[3] V.J. Emery, E. Fradkin, S.A. Kivelson and T.C. Lubensky, Phys. Rev. Lett. 85, 2160 (2000).

[4] A. Vishwanath and D. Carpentier, Phys. Rev. Lett. 86, 676 (2001).

[5] R. Mukhopadhyay, C.L. Kane, T.C. Lubensky, condmat/0102163.

[6] P.W. Anderson, Phys. Rev. Lett. 67, 3844 (1991).

[7] C. S. O'Hern, T. C. Lubensky, J. Toner, Phys. Rev. Lett. 83, 2745 (1999).

[8] J.M. Kosterlitz and D.J. Thouless, J. Phys. C 6, 1181 (1973).

[9] J.P. Rodriguez, P.D. Sacramento, V. Vieira, Phys. Rev. B 56, 13685 (1997)

[10] J.P. Rodriguez, Phys. Rev. B 58, 944 (1998).

[11] M.A. Martin-Delgado, M. Roncaglia, G. Sierra, cond-mat/0101458.

[12] J. Cardy, "Scaling and Renormalization in Statistical Physics", Cambridge University Press (1996).

[13] M. Gunaydin, G. Sierra and P. Townsend, Nucl. Phys. B 253, 573 (1985). 